\title{
HELP: Optimizing Treatment of Parkinson's Disease Patients
}

\author{
Claas Ahlrichs \\ Neusta Mobile Solutions, Software Development, Germany \\ c.ahlrichs@neusta.de
}

Albert Samà

CETpD, Technical Research Centre for Dependency Care and Autonomous Living, Spain

Jordi Rovira Simon
Telefonica I+D, Spain

Simon Herrlich

HSG-IMIT - Institut für Mikro- und Informationstechnik der Hahn-Schickard-Gesellschaft e.V. Germany

Alejandro Rodríguez-Molinero

Fundacion Privada Sant Antoni Abat and Consorci Sanitari del Garraf, Spain

\begin{abstract}
This paper presents a novel health monitoring system for Parkinson's disease (PD) patients called HELP (Home-based Empowered Living for Parkinson's disease patients). The HELP system has been specifically designed and implemented as a health monitoring system in order to optimize treatment and improve quality of life of people with Parkinson's. This is a challenging goal due to the difficulty in establishing a closed-loop system that is able to detect the outcomes of treatment and react accordingly. In a similar way to diabetes treatment where the plasma glucose level can be measured and can be used to regulate drug doses, the HELP system's approach aims to estimate PD symptoms and to adjust the dose of medication in order to reduce symptoms. The proposed health monitoring system is composed of several components: a body sensor \& actuator network managed by a smartphone, a remote monitoring platform for doctors and clinical professionals as well as a telecommunication and service infrastructure. The real advantage derives from having constant medical control without dramatically modifying daily life. The HELP system is going to be evaluated in several cities during the first part of 2012 under daily living conditions with PD patients.
\end{abstract}

Keywords: Parkinson's Disease, Body Sensor and Actuator Network, Health Monitoring System, Ambient Assisted Living

\section{Resumen}

En este trabajo se presenta un nuevo sistema de vigilancia de la salud para pacientes con la enfermedad de Parkinson (EP), pacientes llamados HELP (Fortaleciendo la vida en el hogar de pacientes con la enfermedad Parkinson). El sistema de ayuda ha sido específicamente diseñado e implementado como un sistema de vigilancia de la salud con el fin de optimizar el tratamiento y mejorar la calidad de vida de las personas con Parkinson. Este es un objetivo difícil debido a la dificultad del establecimiento de un sistema de circuito cerrado que es capaz de detectar los resultados del tratamiento y reaccionar en consecuencia. Es una manera similar al tratamiento de la diabetes donde el nivel de glucosa en plasma se puede medir y se puede utilizar para regular las dosis de medicamentos; el enfoque del sistema 
de ayuda tiene por objeto estimar los síntomas de la EP y ajustar la dosis de la medicación con el fin de reducir los síntomas. El sistema de vigilancia de la salud propuesto se compone de varios componentes: un sensor corporal y un actuador de red gestionado por un smartphone, una plataforma de monitorización remota para los médicos y clínicos profesionales, así como el uso de telecomunicaciones y servicios de infraestructura. La verdadera ventaja deriva de que tiene un constante control médico sin modificar drásticamente la vida cotidiana. El sistema HELP va a ser evaluado en varias ciudades durante la primera parte del año 2012 en condiciones de vida diaria con pacientes con EP.

Palabras clave: enfermedad de parkinson, sensor corporal y actuador de red, sistema de vigilancia de la salud, vida asistida por el entorno

\section{Introduction}

In the last decade, advancements in information and communication technologies have given a boost to health monitoring systems. The introduction of electronic health records and digitalization of medical processes have enabled the development of such monitoring systems and telemedicine applications. Nowadays, developed countries use these technologies to reduce the cost of national health systems (e.g. decreasing the length of stay in hospitals, re-occurrence and re-admission rates) (Polisena et al., 2009). Examples of complete health monitoring system are the Phillips Telehealth system (PHILIPS, 2012), the Bosch Health Buddy solution (воSсн, 2012) or the гвм Medical Home solution (IBM, 2012). Several research projects have been developing monitoring systems for diseases such as diabetes (Jia et al., 2011), Congestive Heart Failure (Polisena et al., 2010) and Chronic Obtrusive Pulmonary Disease (Boulos et al., 2009).

Telehealth systems often take advantage of wearable technologies. These technologies include body area networks (BAN) and body sensor and actuator networks (BS \& AN). They are employed to measure and monitor the current state of the user/patient. While BANs can only be used for monitoring purposes, BS \& AN can also be utilized to actuate. Thus actively manipulating the patients state or environment. The same principle is used in systems for diabetes. Here, it is technically possible to measure the plasma glucose level in order to control the basal infusion rate of insulin that is being administrated subcutaneously by a pump (Hovorka, 2006). However, this principle cannot directly be applied to PD as no disease-specific biomarkers (Jankovic,
2008) exist or are simply not practical (e.g. autopsy) (Samii et al., 2004). Currently, the best indicator for drug needs in PD is the quality of motion. Thus, the difficulty to monitor patient's symptoms and infer drug needs arises. However, to the best knowledge of the authors, this principle has not been successfully tested with PD.

The HELP system (Home-based Empowered Living for Parkinson's disease patients) has been designed and implemented as a health monitoring system, which specifically targets the needs of people with Parkinson's. PD is a progressive neurological condition which produces motor symptoms (e.g. rigidity, slowness of movement) and non-motor symptoms (e.g. cognition alterations, sleep disturbances). It is thought to affect more than five million people world-wide (Mathers et al., 2008). It is the fourth most frequent disorder of the nervous system, after epilepsy, brain vascular disease and Alzheimer. The average age at diagnosis is 60 years. Given the rapidly aging population, PD is becoming a major public health issue in Europe (Potocnik, 2008).

The HELP system proposes a solution to improve quality of life of PD patients based on the following three components.

- A BS \& AN consisting of several devices which monitor health parameters (e.g. blood pressure) and body activity (e.g. motor symptoms) as well as administration of drugs.

- A remote point-of-care platform (PoC) that enabled clinical professionals to supervise PD patients.

- A telecommunication and services infrastructure for analyzing and transferring information from the user to the PoC and vice-versa. 
The design deals not only with technological aspects, but it involves medical and social issues as well. HELP aims at filling the gap between these different knowledge domains by using a multidisciplinary, integrated approach derived from the collaboration of partners whose expertise stems from various fields.

The remainder of this paper is organized as follows: section 2 provides insights on clinical aspects of the PD disease; section 3 highlights relevant aspects related to the BS \& AN architecture and PoC. Finally, section 4 draws the conclusions.

\section{Context}

Parkinson's Disease (PD) is a progressive, neurodegenerative disorder of the central nervous system which is characterized by the cardinal symptoms bradykinesia (slow movement), rigidity, resting tremor and postural instability. The cardinal features mainly originate from a dopamine deficiency caused by a degeneration of dopamine producing neurons in part of the brain stem. Without treatment, PD progresses over 5-10 years to a rigid, akinetic state in which patients are no longer capable of caring for themselves.

Despite two centuries since James Parkinson first described the disease (in 1817), the cause of the disease remains unknown. Primarily, therapies aim at minimizing symptoms and maximizing (motor) function as well as quality of life. However, intensive care is required, demanding the allocation of enormous resources besides the strictly medical ones. This leaves room for improvement of disease management and optimizing cost effectiveness of health care plans.

Both pharmacological and surgical treatments are available for PD. The primary treatment is based on medication, while surgical options (e.g. stimulation, ablation) are not suitable for every patient. Thus they can only be applied in specific cases. Levodopa (L-DOPA) is the most effective pharmacological agent for PD and remains the primary treatment for symptomatic patients. However, its long-term use is limited by motor complications and drug-induced dyskinesia (Nutt et al., 1984). Unfortunately, the majority of drugs for treating
PD, particularly L-DOPA, provide pulse stimulation of dopamine release rather than continuous stimulation.

Once the patient has taken the medication, the symptoms relief and they regain control over their movements. Nonetheless due to remaining lack of dopamine production in the basal ganglia, the refilled dopamine does not last forever and motor symptoms are bound to reappear eventually unless another dose of medication is taken. Thus creating fluctuations between periods in which individuals show almost no motor symptoms (known as on period) and periods in which motor symptoms are present (known as off period). In an early stage of the disease, these fluctuations are predictable and cant be almost entirely avoided. However, as the disease progresses the desired effects of levodopa wear off. Thus the effectiveness of medication has a tendency to decrease and the symptoms reappear earlier than before.

With continued progression of the disease, fluctuations between periods of mobility and immobility happen more frequently. In later stages of the disease, the fluctuations become less deterministic and totally unpredictable eventually (ON-OFF phenomenon) (Nutt et al., 1984).

The main issue is to maintain constant dopamine stimulation in order to reduce ON-OFF fluctuations. Keeping a constant level of drugs avoids the dosedependent side effects and co-morbidity, while improving the quality of life of patients. In an indirect way, fewer complications mean reducing possible hospitalizations, thus decreasing medical and assistive costs. Furthermore, if less direct care is needed, their relatives and informal carers will also experience, in an indirect way, an increase of their quality of life, lowering the need to dedicate effort and time to take care of their elderly relatives.

In a more advanced phase of the disease, patients experience freezing episodes in which they actually freeze and are not able to perform any physical activity. Apart from motor symptoms patients can experience non-motor features including depression, sleep disturbances, dizziness, problems with speech, swallowing and sexual functioning. PD greatly impairs the patient's quality of life, increasing also caregiver distress. 
Making the patient's medical data accessible online to medical staff and putting them in contact by means of video conferences, enables a high degree of patient's life comfort, giving them the tranquility to know that their doctor is always aware of their disease situation. When dealing with technological setups that support clinical trials and involve patients, three important aspects must be taken into account: user acceptance, usability and accessibility. All of them have been specifically addressed during the development of the HELP system.

Studies have indicated that drug delivery devices are generally well accepted by patients (La Van, 2003; Curtis et al., 2007). Combing the devices with a transparent and automatic drug calculation ensures a high degree of usability. Medical information of the patient his gathered in an automatic way (e.g. motion detection) or with simple patient intervention (e.g. using a domestic blood pressure meter or answering questionnaires). Thus very little interaction is required by the patients. Furthermore, the interfaces are not dependent on the degree of disability of the person which makes the solution inherently accessible. In addition, the HELP system offers physicians and social care professionals tools to interact with their patients. Their medical and social situation can be handled online and remotely, which makes the HELP system especially accessible for patients with cognitive impairment.

\section{Overview of HELP Monitoring System}

The HELP health monitoring system is composed of several components which can be divided into the following categories: (1rst) a network of sensors and actuators being worn by the patient, (2nd) a remote monitoring platform being used by doctors and clinical professionals as well as (3rd) a telecommunication and services infrastructure. All of which are described in the following subsections. The real advantage of the HELP system derives from having the constant medical control necessary for a PD patient without dramatically modifying his/her daily life.

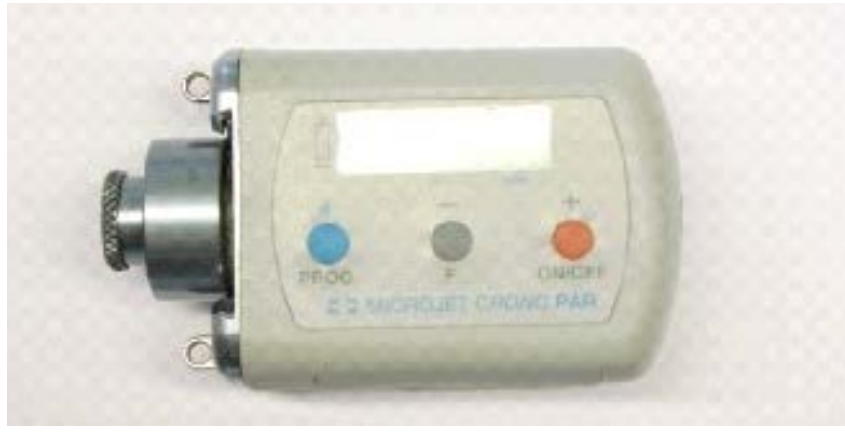

Figure 1: The subcutaneous pump is used for $d y$ namic drug delivery. The pump has been modified to receive commands via a ZigBee interface

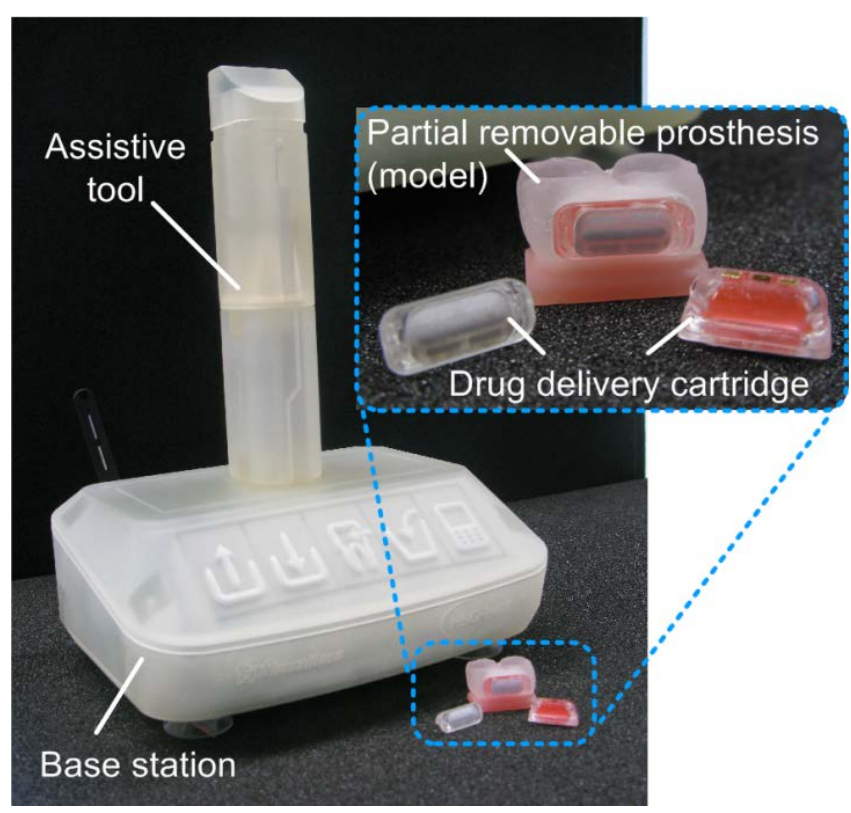

Figure 2: The intra-oral cartridge is used for constant drug delivery. The base station has been equipped with a ZigBee communication module

\section{Body Sensor and Actuator Network}

The BS \& AN consists of two sensors, one actuator (either a subcutaneous pump or an intra-oral drug delivery device) and a smartphone. In general, both sensors provide information on the activity and drug needs of the patient. Based on this information the required amount of drug is determined and eventually administered to the patient by the actuator. The smartphone acts as a control station for the patient. It collects sensor information and adjusts the actuators. All five components are highlighted and briefly described in Figure 1. 
The actuator provides the means of delivering medication to the patient. A subcutaneous pump and an intra-oral drug delivery device cover both a dynamic (advanced stage of disease) and constant drug delivery (early and moderate stages of disease). The subcutaneous pump (see Figure 1) is worn by the patient and capable of dynamically delivering medication. It can be remotely controlled in order to adjust the rate of infusion. A constant delivery of medication is taken care of by the intra-oral device (see Figure 2). While the intra-oral device and the base station are new developments, the subcutaneous pump is a modified version of a commercially available device which is extended by a wireless communication module based on the ZigBee communication standard.

A miniaturized cartridge is capable of delivering drugs according to the patient needs, in periods lasting days, weeks or months. An osmotic pumping principle uses saliva to release highly precise a separately stored drug to the buccal mucosa (Herrlich et al., 2011), thus avoiding first pass metabolism and drug plasma level fluctuations. The disposable cartridge is magnetically attached into a receptacle of a partial removable prosthesis and can be replaced by the patient himself.

The concept envisages varying the patient's medication within boundaries specified by medical professionals. Relevant information like patient adherence to the therapy, medication and delivered amount of the cartridge can be determined by readout of its fill level, when placed on the base station.
The sensors provide measurements with which the patient's state is estimated. A blood pressure monitor is used to supervise the overall health condition, whereas an inertial sensor provides relevant information about the physical status (e.g. quantity of movement, motor symptoms and on/ off state). Information of the latter kind is used to infer drug needs. The inertial sensor is embedded into a device that offers enough computational capabilities to process data in real-time and has been designed to be used for long periods of time. The inertial body sensor is a single unit device which is encapsulated in a $78 \times 37 \times 10 \mathrm{~mm}$ black case. It weights $125 \mathrm{~g}$ (battery included). The sensor also has an embedded ZigBee module for wireless communication.

Internally, the device includes a micro-controller that handles the sub-systems (see Figure 3). The micro-controller provides the system with the capacity to acquire, order, compute and send data while handling other functions like energy management and user interface supervision.

The smartphone is a commercially available mobile device and holds a custom application as well as a special MicroSD card with an embedded ZigBee node. It is in charge of the management of the sen- 


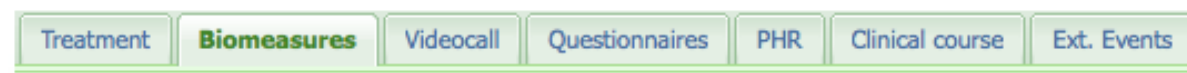

Last value

Blood Pressure Progress

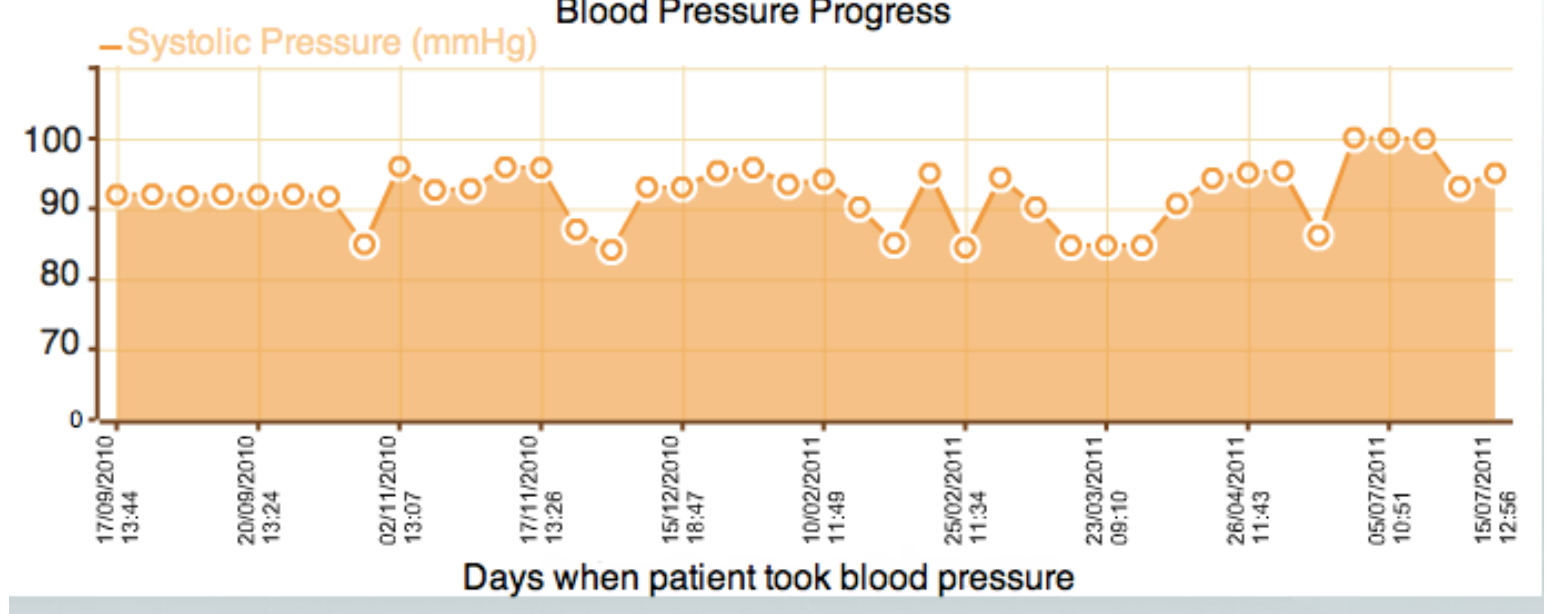

Figure 4: Illustrates the user interface provided to clinical professionals sors and actuators described above. It is also used to forward information gathered by the devices in the BS \& AN to the PoC. The smartphone has higher processing and memory capabilities with respect to sensor nodes and it can be used to 1) configure, monitor and control the sensor network, 2) collect and send data to a remote service center, and 3) locally process data (e.g. to manage alarms to be sent to the service center).

The smartphone is connected to the other devices by means of a ZigBee network and can be considered the sink (or the coordinator, following the ZigBee terminology) of a classical star topology network. In particular, the intra-oral device communicates to the smartphone when it is placed on the base station, as it would be unfeasible with the current technology to implant a similar radio interface directly inside the cartridge.

The setup of the network must also ensure security features, hence the communication between medical devices and smartphone is encrypted and point-to-point. In order to avoid that external devices access sensitive data, the smartphone is instructed to establish a communication only with its own system devices. Moreover, each transmission contains the patient ID, the device ID and the time and date of transmission.

\section{Point of Care Platform}

The platform, that is being described in this subsection, is intended to be used by doctors and other clinical professionals. It shows information on the patient's state (see Figure 4) and enables to review and adjust the treatment of each patient. A rule engine ( $\mathrm{RE}$ ) system processes data coming from sensors and provides an estimation of the patient status. Furthermore, the drug needs are estimated. Both components are described in more details below.

The RE system suggests the amount of drug to be delivered to the patient. A clinician will review the drug dose suggestion and confirm or reject it. The drug dose calculation is estimated by using two input signals: information provided by the sensors and assignments provided by the physicians via PoC (a set of thresholds specific to each patient).

Important figures are graphically represented (e.g. blood pressure, patient's activity, number of steps, 
drug dose), so doctors can make wiser decisions (e.g. calling a relative if something looks strange or adjusting the drug dose). Furthermore, the PoC provides an interface for viewing alarms (e.g. patient has fallen, drug dose needs to be adjusted, etc.).

The RE provides the service to diagnose a patient's status based on a set of medical rules, which are supplied by clinical professionals and medical partners. Each medical rule has a condition, a result or consequence and a frequency. Once a patient is found that matches a rule's condition the result (e.g. raise an alarm) is applied. The conditions may have varying complexities which range from simple comparisons (e.g. equal, greater than, etc.) and mathematical operations (e.g. addition, division, etc.) to logic inference (e.g. conjunction, disjunction, etc.). The consequences are not limited to but usually raise an alarm which is then reviewed by medical professionals.

The frequency of a medical rule specifies how often it is checked. Rules with a high priority are checked up to every minute whereas rules with a lower priority might only be checked every 8 hours. The RE periodically checks all rules according to their specified frequencies. The central database is queried for new patient information and the rules are applied to it. The RE can easily handle thresholds or settings specific to each patient.

\section{Telecommunication and Services Infrastructure}

The telecommunication infrastructure ties the previously mentioned components together. It provides the means to exchange data with the central server. Furthermore, the envisioned infrastructure also enables videoconferencing between a patient and relatives, social caretakers and doctors.

The smartphone transmits the latest patient data to the server and retrieves the inferred drug needs. The latest patient data is collected from the inertial sensor and blood pressure monitor, whereas the drug needs are forwarded to the subcutaneous pump where the amount of delivered drug is adjusted.

The PoC retrieves the latest patient data from the server and displays them to medical personnel. Doctors have the opportunity to accept or reject changes in drug needs based on the provided data. Furthermore, the therapy for each patient can be adjusted.

\section{Conclusions}

The paper has provided insights on the HELP system, detailing the system architecture and the Bs \& AN created around a smartphone and medical devices. The whole system provides clinicians a telehealth tool for monitoring and treating PD in real-time. Help is envisioned to fulfill the so far unmet needs derived from the drawbacks of traditional PD treatments. Thus, it would improve the quality of life of PD patients and reduce co-morbidity, and by doing so, enable the users to conduct an independent life at their homes.

The advantages of HELP will be validated during the last phase of the project. It will include trials with PD patients, which will provide the necessary feedback to assess the consistency and efficiency of the HELP solution and its compliance with such challenging objectives.

The development of the presented equipment implies the confluence of very different competences, and may give the desired precise control while offering the most efficient therapeutic outcomes. By validating the HELP system and demonstrating an increase in the quality of life of PD patients, this kind of innovative high-tech system may become a new commercial challenge for pharmaceutical and telehealth companies.

\section{Acknowledgements}

The authors would like to acknowledge all the other partners of the HELP-ALL1 Consortium, for their support in preparing the paper. 


\section{References}

BOSCH (2012). Health buddy system. www.bosch-telehealth.com.

Boulos, M. N. K., Lou, R. C., Anastasiou, A., Nugent, C. D., Alexandersson, J., Zimmermann, G., Cortes, U. and Casas, R. (2009). Connectivity for healthcare and well-being management: Examples from six European projects. Int. J. Environ. Res. Public Health, 6(7): 1947-1971.

Curtis, D. C., Linder, V. and Sia, S. K. (2007). Lab-on-a-chip devices for global health: Past studies and future opportunities. Lab Chip, 7: 41-57.

La Van, D. A., Mc Guire, T. and Langer, R. (2003). Small-scale system for in vivo drug delivery. Nature Biotetechnology, 21: 1184-1191.

J. Potocnik (2008). A focus on Parkinson's disease and the European society. 1st European Brain Policy Forum, Brussels.

Herrlich, S., Lorenz, T., Marker, M., Spieth, S., Messner, S. and Zengerle, R. (2011). Miniaturized osmotic pump for oromucosal drug delivery with external readout station. In Proc. of IEEE EMBC, 8380-8383.

Hovorka, R. (2006). Continuous glucose monitoring and closed-loop systems. Diabetic Medicine, 23(1): 1-12.

IBM (2012). Patient-centered medical home. www.ibm.com/healthcare/medicalhome.

Polisena, J., Tran, K., Cimon, K., Hutton, B., McGill, S., Palmer, K. and Scott, R. E. (2010). Home telemonitoring for congestive heart failure: a systematic review and meta-analysis. J Telemed Telecare, 16(2): 68-76.

Samii, A., Nutt, J. G., and Ransom, B. R. (2004). Parkinson's disease. The Lancet, 363(9423): 1783-1793.

Jankovic, J. (2008). Parkinsons disease: clinical features and diagnosis. Journal of Neurology, Neurosurgery \& Psychiatry, 79(4): 368-376.

Jia, H., Feng, H., Wang, X., Wu, S. S. and Chumbler, N. (2011). A longitudinal study of health service utilization for diabetes patients in a care coordination home-telehealth programme. J Telemed Telecare, 17(3): 123-126.

Mathers, C., Fat, D. M., Boerma, J. T. and Organization., W. H. (2008). The global burden of disease: 2004 update. World Health Organization, Geneva, Switzerland.

Nutt, J. G., Woodward, W. R., Hammerstad, J. P. Carter, J. H. and Anderson, J. L. (1984). The on-off phenomenon in Parkinson's disease. New England Journal of Medicine, 310(8): 483-488.

PHILIPS (2012). Healthcare at home. www.healthcare.philips.com.

Polisena, J., Coyle, D., Coyle, K. and McGill, S. (2009). Home telehealth for chronic disease management: A systematic review and an analysis of economic evaluations. International Journal of Technology Assessment in Health Care, 25: 339-349. 